\title{
Direct observation of neutrino oscillations at the Sudbury Neutrino Observatory
}

\author{
B. Ananthanarayan and Ritesh K. Singh
}

The Sudbury Neutrino Observatory (SNO) located in Canada, has recently announced evidence for the direct observation of oscillation of neutrino flavours ${ }^{1 \mathrm{a}}$. The observations indicate that during the flight from the interior of the sun to the earth, the neutrinos produced as electrontype neutrinos change their flavour. Neutrinos are fundamental particles that come in three types (flavours): electron, muon and tau denoted by $v_{\mathrm{e}}, v_{\mu}$ and $v_{\tau}$ respectively. Electron-type (anti)neutrinos are produced, for instance, in radioactive decays of certain heavy nuclei, and are also produced copiously in stars during the process of nucleosynthesis. It may be recalled that a long-standing problem, called the 'solar neutrino problem', is associated with the shortfall in the number of neutrinos observed on the earth compared to the number expected from calculations based on the standard solar model and the standard model of elementary particle physics. The solar models happen to be stringently constrained and the flux of neutrinos produced in the sun is known to practically no uncertainty, due to extreme sensitivity of the flux to the values of the parameters going into the solar model. As a result, it has been long believed that any solution to the problem must come from the elementary particle physics sector, such as the oscillation of flavours, or the possible interaction of the neutrinos with the solar magnetic fields which might significantly scatter away the neutrinos that were directed towards the earth. A flavour oscillation necessarily implies that the neutrinos are massive particles, and was first pointed out a long time ago by the Italian-born Soviet scientist, Bruno Pontecorvo. Whereas there are constraints on the masses of the electron, muon and tau type neutrinos $\left(m_{v_{\mathrm{e}}} \leq 2.8 \mathrm{eV} / \mathrm{c}^{2}, m_{v_{i j}} \leq\right.$ $170 \mathrm{keV} / \mathrm{c}^{2}$ and $\left.m_{v_{\mathrm{U}}} \leq 18.2 \mathrm{MeV} / \mathrm{c}^{2}\right)$, the oscillation can, in principle probe differences of the squares of the masses, and the probability of conversion from one flavour into another depends on the path length between production and observation, and on the kinetic energy of the neutrino that is being observed. The observation from the SNO definitively shows that the electron-type neutrino oscillates into an admixture of the other two flavours. The SNO collaboration also reports a possible day-night effect ${ }^{\mathrm{lb}}$, in that there seems to be some indication that the probability of conversion of the electrons depends on the path length, which depends on whether the observation is made during the day or night.

The experiment is based on the principle of detecting interactions of neutrinos with matter in an ingenious manner, which uses heavy water as the medium in which the interactions take place and in which the reaction products are detected. This differs from earlier measurements which used normal water, at Kamiokande and the super-Kamiokande experiments, and from those based on radiochemical measurements which used interactions of the neutrinos with nuclei of chlorine in carbon tetrachloride or with gallium nuclei. For completeness, we note that the solar neutrino problem was first established by the historic chlorine experiment of Ray Davis at the Homestake Mine in South Dakota, USA, which employs the following reaction to detect neutrinos:

$$
{ }^{37} \mathrm{Cl}+v_{\mathrm{e}} \rightarrow{ }^{37} \mathrm{Ar}+e^{-} .
$$

The gallium experiments use the reaction

$$
{ }^{71} \mathrm{Ga}+v_{\mathrm{e}} \rightarrow{ }^{71} \mathrm{Ge}+e^{-} .
$$

The neutrinos that are observed at the SNO are produced in the core of the sun in the following reaction:

$$
{ }^{8} \mathrm{~B} \rightarrow{ }^{8} \mathrm{Be}+e^{+}+v_{\mathrm{e}} .
$$

The kinetic energy of the neutrinos produced in this reaction could vary from as little as a fraction of an $\mathrm{MeV}$ to approximately $15 \mathrm{MeV}$. These neutrinos interact with the water molecules in a variety of ways. The normal water detector detects this neutrino from its elastic scattering (ES) with the electrons in the water molecules. The ES measurements are predominantly sensitive to the electron-type flavour only. The measurements of superKamiokande had established from the event rates observed, a shortfall in the expected event rate, consistent with the earlier radiochemical measurements which had led to the classical solar neutrino problem. These observations were based on the charged current (CC) reactions. The full electroweak theory of Glashow, Salam and Weinberg has established that the theory also has, in addition to the conventional electromagnetic interaction, what are called the neutral current (NC) interactions. All the interactions arise from the exchange of (virtual) intermediate vector bosons; the $\mathrm{CC}$ interactions arise from $W^{ \pm}$, the NC interactions arise from a neutral boson called $Z^{0}$, just as the electromagnetic interactions arise from the photon. Here we note that the main principle in both the normal and heavy-water detectors for the observation of the scattered electron in the ES or the produced electron in the $\mathrm{CC}$ reaction is that of the detection of Cerenkov light produced by the ultrarelativistic electron during its motion with its velocity exceeding that of light in the medium (water). The Cerenkov light is detected by photomultiplier tubes at the boundary of containers. Note also that the water has to be of extraordinary purity in order to prevent attenuation of the light during its travel from the electron source to the detector. The remarkable advantage of the heavy-water detector is its capability to observe the neutral current interactions as well, in addition to measuring certain other $\mathrm{CC}$ interactions which we will describe below. The measurements of the ES and CC reaction rates by the SNO collaboration were reported earlier ${ }^{1 c}$, which further confirmed the solar neutrino problem.

The remarkable advantage of the heavywater detector is its capability to observe the NC interactions as well, in addition to observing the $\mathrm{CC}$ interactions. It must be mentioned that the construction of the SNO experiment was directly inspired by an important paper by the late Herb $\mathrm{Chen}^{2}$. The crucial property of the heavywater detector arises from the fact that the deuterium nucleus has a remarkably small binding energy of $2.225 \mathrm{MeV}$. This may be contrasted with the typical binding energies of 8-9 MeV/nucleon for most 
nuclei. As a result, the kinetic energy of the neutrinos is sufficiently large so as to induce the following reactions

$$
\begin{aligned}
& v_{\mathrm{e}}+{ }^{2} \mathrm{D} \rightarrow e^{-}+2 p \\
& v_{\mathrm{l}}+{ }^{2} \mathrm{D} \rightarrow v_{\mathrm{l}}+p+n, l=v, \mu, \tau
\end{aligned}
$$

The reason why the CC reaction above is sensitive only to the electron-type neutrino is that kinetic energy of the neutrinos produced in the boron reaction is sufficient only to produce electrons $\left(m_{\mathrm{e}}=\right.$ $0.511 \mathrm{MeV} / \mathrm{c}^{2}$ ), in accordance with Einstein's mass-energy equivalence, whereas the muons and the tau-leptons are too massive to be produced in this reaction $\left(m_{\mu}=105.7 \mathrm{MeV} / \mathrm{c}^{2}, m_{\tau}=1777 \mathrm{MeV} / \mathrm{c}^{2}\right)$. The NC reaction does not have this kinematic constraint, and is therefore sensitive to all flavours. The combination of the small binding energy of the deuterium nucleus with the $\mathrm{NC}$ reaction is capable of producing a characteristic signal which can be detected when the deuterium nucleus is shattered and the neutron is liberated. Nevertheless, the heavywater detector observed the $\mathrm{CC}$ reaction above and the ES reaction rates by 2001 , but had to wait until 2002 to observe the NC. The latter required that ultra-pure common salt $(\mathrm{NaCl})$ be introduced into the heavy water so that the neutrons produced in the $\mathrm{NC}$ reactions could be absorbed by the $\mathrm{Cl}$ nuclei, and then pro- duce a characteristic $8.6 \mathrm{MeV}$ gamma-ray signal. Otherwise neutrons were detected by characteristic $6.25 \mathrm{MeV}$ gamma ray when they get absorbed by deuterium in heavy water. The neutron absorption probability in heavy water is about $25 \%$, which increased to $85 \%$ by addition of ultra-pure $\mathrm{NaCl}$. This was achieved and the results subsequently reported ${ }^{1 \mathrm{a}}$. The final numbers quoted therein translate to $2 / 3$ of the electron-type neutrinos oscillating into muon- and tau-type flavours. Furthermore, the observation of a nonvanishing day-night effect shows that there may be some regeneration of the electron-type neutrino flux in the passage of the neutrinos from the sun through the earth. Such an effect, known as the Mikheyev-Smirnov-Wolfenstein effect, has been studied in the past and is now likely to be constrained quite effectively, or alternative vacuum oscillation scenarios are likely to be constrained as well. For a recent discussion of the impact of the SNO measurements on theoretical scenarios, see Bahcall et al. ${ }^{3}$.

It must be mentioned again that the advantages of heavy water also leads to the possibility of large backgrounds. In fact, the SNO experiment is located deep underground in nickel mines in Canada, and the heavy water which is stored in a large acrylic container, is also surrounded by jackets containing normal water in order to absorb radiation from the surrounding rock and also from cosmic ray sources which could easily generate spurious signals which serve as a background.

In conclusion, we note that the remarkable experiment at the SNO based on the deep insights of Chen has resolved the solar neutrino problem in favour of a solution arising from neutrino oscillations, rather than from unknown inadequacies of the standard solar model. The SNO collaboration is expected to improve its statistics and bring down uncertainties in its measurements, and will pave the way to confirming and constraining theoretical scenarios which account for neutrino oscillations.

1. a. Ahmad, Q. R. et al., [SNO Collaboration], Phys. Rev. Lett., 2002, 89, 011301; b. 2002, 89, 011302; c. 2001, 87, 071301.

2. Chen, H. H., Phys. Rev. Lett., 1985, 55, 1534.

3. Bahcall, J. N., Gonzalez-Garcia, M. C. and Pena-Garay, C., arXiv:hep-ph/0204314.

B. Ananthanarayan* and Ritesh K. Singh are in the Centre for Theoretical Studies, Indian Institute of Science, Bangalore 560012 , India. *For correspondence (e-mail:anant@cts.iisc.ernet.in).

\section{COMMENTARY}

\section{Auto fuel policy: a question of balance}

\section{Uday T. Turaga}

The Mashelkar Committee's interim report ${ }^{1}$ on auto fuel policy (see Box 1 for a chronology of key events) has created intense controversy, blurring the key issues at stake. Environmentalists consider the report as a sell-out to the so-called diesel lobby, ensuring India's 'return to the era of smoke and fumes'. As a fuel scientist at The Pennsylvania State University, specializing in the removal of sulphur from diesel fuel, I write this to present a different perspective.

The Mashelkar report, surprising as it may be in these frenzied times, is one of the few good pieces of Indian public policy produced in recent times. It is stupefying that key fuel technology and policy players like the oil refineries, automakers, research institutes like the Indian Institute of Petroleum, and the Indian government have, as always, blundered by refusing to react, let alone defend the Mashelkar report against the misplaced fury of the environmentalists' articulate and high-profile media campaign.

\section{Super emitters limit leapfrogging advantages}

At the core of the controversy is the Mashelkar report's timeframe for phasing in fuel specifications. The Mashelkar report suggests that:
(1) Euro II regulations be extended no later than 2003 to Bangalore, Hyderabad and Ahmedabad in addition to the four metros they are currently active in.

(2) Euro III (diesel fuel with $50 \mathrm{ppm}$ sulphur) regulations be enforced in these seven mega cities and the entire nation by April 2005 and 2010, respectively.

(3) Euro IV be evaluated for economic feasibility.

The green lobby calls these 'weak', 'incremental' measures and instead desires that India leapfrog to Euro III or IV fuel specifications immediately which alone will, in their view, reduce health hazards. 